\title{
Compositional Plane of a New Wide-Gap Solid Solution Semiconductor CaPbSeS and Epitaxial Thin Film Growth of CaSe
}

\author{
Seishi Abe and Katashi Masumoto \\ The Research Institute for Electric and Magnetic Materials, Sendai 982-0807, Japan
}

\begin{abstract}
We have systematically investigated the compositional plane of solubility range and lattice constants in $\mathrm{Ca}_{1-x} \mathrm{~Pb}_{x} \mathrm{Se}_{1-y} \mathrm{~S}_{y}$ system, and as a first step for preparation of the ternary system, we have also investigated the growth and characterization of the CaSe thin films.

Solubility range and lattice constant of a $\mathrm{Ca}_{1-x} \mathrm{~Pb}_{x} \mathrm{Se}_{1-y} \mathrm{~S}_{y}$ system was investigated using powder synthesis under thermal equilibrium condition. A CaSe thin film was grown on a cleaved $\mathrm{BaF}_{2}(111)$ substrate by means of a hot-wall epitaxy.

The solubility limit at $1273 \mathrm{~K}$ varies with respect to the Se concentration $y$, taking a minimum limit of 0.04 at $y=0.8$ and a maximum of 0.24 at $y=0$. It is found that the system can be lattice-matched to $\mathrm{PbS}$ and InP. The CaSe thin films are grown epitaxially at substrate temperature range between 673 and $873 \mathrm{~K}$. The energy band gap of the film is estimated to be $4.62 \mathrm{eV}$ at RT through measurement of transmittance and reflectance of the film, having full-width at half-maximum (FWHM) of X-ray rocking curve of $0.08^{\circ}$ by $\omega-2 \theta$ scan at (222) Bragg reflection.
\end{abstract}

(Received March 22, 2005; Accepted June 6, 2005; Published September 15, 2005)

Keywords: solid solutions, calcium compounds, semiconducting II-VI materials, heterojunction semiconductor devices, hot-wall epitaxy, compositional plane, epitaxial thin film growth

\section{Introduction}

Novel devices such as quantum cascade laser and optical switch can be operated through intersubband transition of electron in superlattice structure, which is made of alternated layers of semiconductors with different energy band gaps. Enlargement of the conduction band offset in heterojunction allows to shorten the wavelength of the emitted light toward near-infrared region for quantum cascade laser, and to apply the optical switch as an advanced device in the optical communication system at around $1.55 \mu \mathrm{m}$ used practically.

Up to now, many studies for the layered semiconductors with different energy band gaps have been carried out on $\mathrm{AlN} / \mathrm{GaN},{ }^{1)} \mathrm{AlAs} / \mathrm{InGaAs},{ }^{2}{ }^{2} \mathrm{AlAsSb} / \mathrm{InGaAs}^{3)}$ and $\mathrm{BeTe} /$ $\mathrm{ZnSe}{ }^{4)}$ In addition to the consideration of band alignment, lattice-match condition between such heterostructure and substrate is also important, since the large lattice mismatch between AlAs and InP substrate in the AlAs/InGaAs system, for example, either limits the number of quantum wells that can be grown without significant material degradation or requires a novel growth technique of growing a linearly graded composition buffer layer to accommodate the strain. ${ }^{2)}$ Accordingly, AlAsSb/InGaAs heterostructure can only be lattice-matched to substrate among these systems, bringing conduction band offset of $1.75 \mathrm{eV}^{5,6)}$ under type II band alignment in spite of energy band gap difference of $1.8 \mathrm{eV}$ between the two systems.

In order to shorten the intersubband transition wavelength toward visible region, other materials with larger conduction band offset should be designed under keeping latticematched condition. As another possible candidate for such materials, we propose a new wide-gap solid solution of $\mathrm{Ca}_{1-x} \mathrm{~Pb}_{x} \mathrm{Se}_{1-y} \mathrm{~S}_{y}$ system. This system is characterized with the combination of wide gap calcium chalcogenide systems of $\mathrm{Ca}_{1-x} \mathrm{~Pb}_{x} \mathrm{Se}_{1-y} \mathrm{~S}_{y}$ and narrow gap lead chalcogenide systems of $\mathrm{Pb}_{1-x^{\prime}} \mathrm{Ca}_{x^{\prime}} \mathrm{S}_{1-y^{\prime}} \mathrm{Se}_{y^{\prime}}$. The latter system has been found for a mid-infrared laser in our previous study. ${ }^{7)}$ It is expected in the proposed system that there is a considerable large difference in the energy band gap between the two systems, and that its layered structure is lattice-matched to substrate. As a result of the design of the new material, $\mathrm{Ca}_{1-x} \mathrm{~Pb}_{x} \mathrm{Se}_{1-y} \mathrm{~S}_{y}$ system has potential to bring larger conduction band offset compared to the AlAsSb/InGaAs heterostructure among the lattice-matching systems, thanks to these materials combination of wider- and narrowersemiconductors in energy band gap.

The main advantage of $\mathrm{CaSe}$ in the alkaline-earth selenide compounds is followed that $\mathrm{CaSe}$ is predicated to be direct band-gap material since the valence-band maximum occurs at the $\Gamma$ point whereas the minimum in conduction band at the $\mathrm{X}$ point for $\mathrm{MgSe}$, at the $\Gamma$ point for $\mathrm{CaSe}$, and at the $\mathrm{K}$ point for $\mathrm{SrSe}{ }^{8)}$

The fundamental problem may be difficulty to predict the properties of the solid solution $\mathrm{Ca}_{1-x} \mathrm{~Pb}_{x} \mathrm{Se}_{1-y} \mathrm{~S}_{y}$ at a given composition $x$ and $y$, since a mixed crystal often has a miscibility gap. And besides, the compositional plane of the system will be valuable to design each of the layers in the lattice-matched hetero-structure. On the other hand, a method to prepare ternary or quaternary thin films must be developed in order to fabricate hetero-epitaxial superlattice structure employing the $\mathrm{Ca}_{1-x} \mathrm{~Pb}_{x} \mathrm{Se}_{1-y} \mathrm{~S}_{y}$ system. However, even the binary CaSe epitaxial thin film has not been fabricated yet, and also, the energy band gap at RT of CaSe is not still clear. Therefore, in the present study, we have systematically investigated the compositional plane of solubility range and lattice constants in $\mathrm{Ca}_{1-x} \mathrm{~Pb}_{x} \mathrm{Se}_{1-y} \mathrm{~S}_{y}$ system using powder synthesis, and as a first step for preparation of the ternary system, we have also investigated the growth and characterization of the CaSe thin films using hot-wall epitaxy method.

\section{Experimental Procedure}

Solubility range and lattice constant of $\mathrm{a} \mathrm{Ca}_{1-x} \mathrm{~Pb}_{x} \mathrm{Se}_{1-y} \mathrm{~S}_{y}$ system was investigated using powder synthesis under 


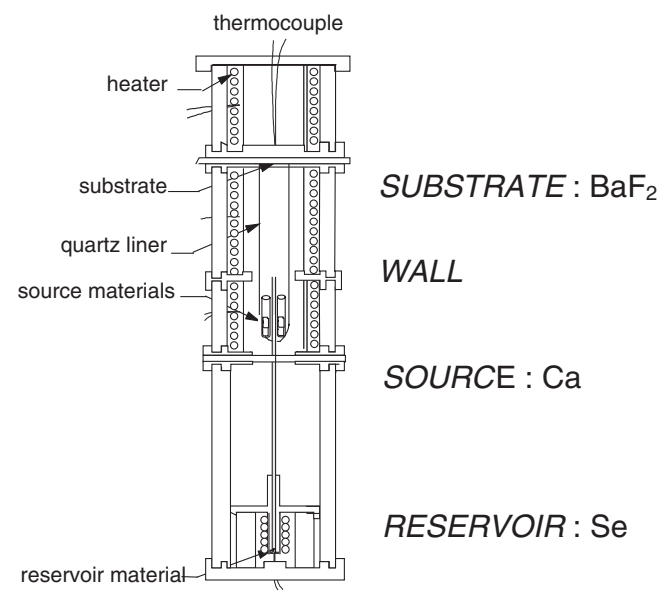

Fig. 1 Schematic representation of the hot-wall epitaxy apparatus used for the fabrication of CaSe thin films.

thermally equilibrium condition. The elements used were $\mathrm{CaSe}(99.5 \%), \mathrm{CaS}(4 \mathrm{~N}), \mathrm{PbS}$ and $\mathrm{PbSe}$, and they were weighed for a desired composition of $\mathrm{Ca}_{1-x} \mathrm{~Pb}_{x} \mathrm{Se}_{1-y} \mathrm{~S}_{y}$, and mixed in an agate mortar. Then, the mixed powder was heattreated at $1273 \mathrm{~K}$ for $48 \mathrm{~h}$ in order to obtain a thermal equilibrium state. The above-mentioned $\mathrm{PbS}$ and $\mathrm{PbSe}$ compounds were synthesized from the elements having $6 \mathrm{~N}$ purity. The lattice constants of the $\mathrm{Ca}_{1-x} \mathrm{~Pb}_{x} \mathrm{Se}_{1-y} \mathrm{~S}_{y}$ system thus obtained were determined by powder X-ray diffraction with $\mathrm{Cu} \mathrm{K} \alpha$ radiation, and then the solubility range was estimated.

A CaSe thin film was grown on cleaved $\mathrm{BaF}_{2}(111)$ substrates by means of a hot-wall epitaxy (Fig. 1). The choice of $\mathrm{BaF}_{2}$ substrate was motivated by its ultraviolet-visible transparency, negligible vapor pressure at a grown temperature employed and relatively near lattice constant for $\mathrm{CaSe}$ compared to other substrate. The apparatus used consists of four electric furnaces, which are for substrate, wall, source and reservoir, as shown in the figure, and can be controlled independently in temperature. $\mathrm{Ca}(99.5 \%)$ is used as the source material, and $\mathrm{Se}(6 \mathrm{~N}$ pure) is used as the reservoir material. Each of the vaporized elements would react on the substrate to form CaSe compound.

Before the growth of CaSe thin film, Ca was preheated at a source temperature $\left(T_{\text {sou }}\right)$ of $843 \mathrm{~K}$ for $30 \mathrm{~min}$ in order to stabilize the $\mathrm{Ca}$ vapor flux through the growth run. Wall temperature in the apparatus was kept constant at $823 \mathrm{~K}$ through the present study.

Growth orientation of the films was determined by highresolution X-ray diffraction (XRD) with a four-crystal monochromator. Thickness of the films was measured by a surface texture analysis system. Spectral transmittance and reflectance at RT of the films were observed at the wavelength range from 600 to $200 \mathrm{~nm}$ by UV/VIS and NIR measurement.

\section{Results and Discussions}

Figure 2 shows the typical powder X-ray diffraction patterns of the $\mathrm{Ca}_{1-x} \mathrm{~Pb}_{x} \mathrm{Se}_{1-y} \mathrm{~S}_{y}$ solid solution system $(y=$ $0)$. As seen in the figure, a single phase in the $\mathrm{Ca}_{1-x} \mathrm{~Pb}_{x} \mathrm{Se}$

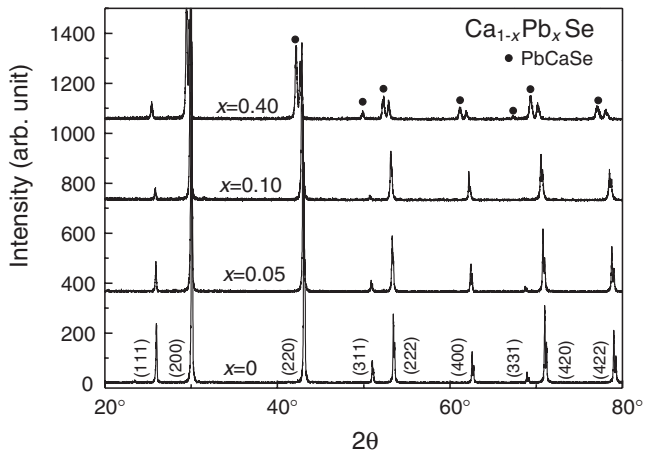

Fig. 2 Typical powder X-ray diffraction patterns of the $\mathrm{Ca}_{1-x} \mathrm{~Pb}_{x} \mathrm{Se}_{1-y} \mathrm{~S}_{y}$ $(y=0)$ solid solution system.

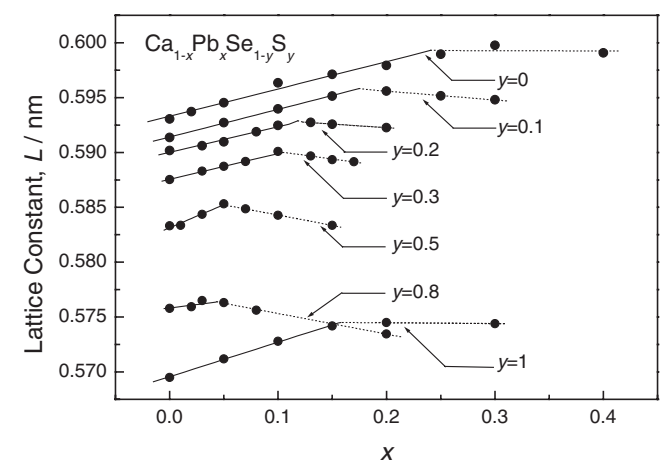

Fig. 3 Lattice constants of the $\mathrm{Ca}_{1-x} \mathrm{~Pb}_{x} \mathrm{Se}_{1-y} \mathrm{~S}_{y}$ system as a function of $x$.

system is observed on the concentration range below $x=0.1$, which can be characterized with $\mathrm{NaCl}$ structure, while two phases consisting of $\mathrm{Ca}$ rich $\mathrm{Ca}_{1-x} \mathrm{~Pb}_{x} \mathrm{Se}$ compound and $\mathrm{Pb}$ rich $\mathrm{Pb}_{1-x^{\prime}} \mathrm{Ca}_{x^{\prime}}$ Se compound are observed at $x=0.4$.

Figure 3 shows the lattice constants of various $\mathrm{Ca}_{1-x^{-}}$ $\mathrm{Pb}_{x} \mathrm{Se}_{1-y} \mathrm{~S}_{y}$ system as a function of $x$ at the fixed composition $y$, where the results of the $\mathrm{Ca}$ rich region are represented. A lattice constant of the $\mathrm{Ca}_{1-x} \mathrm{~Pb}_{x} \mathrm{Se}_{1-y} \mathrm{~S}_{y}$ system shown in the figure were determined by linear extrapolation to zero of the values derived through the Nelson-Riley function ${ }^{9)}$ employing the Bragg peaks above $2 \theta=100^{\circ}$. The solid line indicates the single-phase region, and the dotted line indicates the two phases region, in which the lattice constants of the $\mathrm{Ca}$ rich compound are presented. As can be seen in the figure, the lattice constants increase linearly in proportion to $x$ within the solubility range, irrespective of the concentration $y$. The on-setting composition $x$ to deviate from the linearity according to Vegard's law ${ }^{10)}$ is always regarded as the solubility limit. In the two phases region, on the other hand, the lattice constants at $y=0$ and 1.0 seems to be almost constant with respect to $x$ in contrast to decrease in lattice constant with increasing $x$ for other concentrations $y$. These different variations of the lattice constants in the two phases region is attributed to difference in the number of degree of freedom in the $\mathrm{Ca}_{1-x} \mathrm{~Pb}_{x} \mathrm{Se}_{1-y} \mathrm{~S}_{y}$ system according to Gibbs' phase rule. Namely, in case of solid-solid reaction, the number of degrees of freedom must be specified to completely describe the state of the system at $y=0$ and 1.0 when the temperature of heat-treatment is kept constant at $1273 \mathrm{~K}$ because of the component of 2 in the ternary system, 
Table 1 Lattice constants in the solubility range as a function of $x$ for $\mathrm{Ca}_{1-x} \mathrm{~Pb}_{x} \mathrm{Se}_{1-y} \mathrm{~S}_{y}$.

\begin{tabular}{lcl}
\hline$y$ & $\begin{array}{c}\text { Lattice constant } \\
(\mathrm{nm})\end{array}$ & \\
\hline 1 & $0.5696+0.0314 x$ & $x \leq 0.16$ \\
0.8 & $0.5757+0.0213 x$ & $x \leq 0.04$ \\
0.5 & $0.5832+0.0366 x$ & $x \leq 0.05$ \\
0.3 & $0.5876+0.0235 x$ & $x \leq 0.10$ \\
0.2 & $0.5900+0.0234 x$ & $x \leq 0.12$ \\
0.1 & $0.5914+0.0252 x$ & $x \leq 0.18$ \\
0 & $0.5933+0.0249 x$ & $x \leq 0.24$ \\
\hline
\end{tabular}

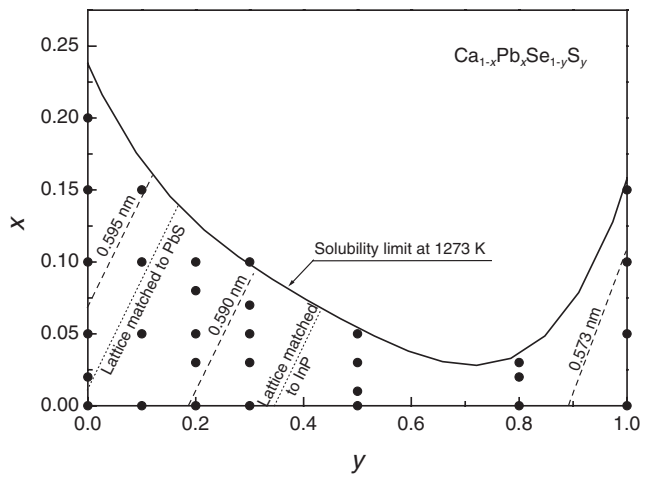

Fig. 4 Solubility range and lattice constant of the $\mathrm{Ca}_{1-x} \mathrm{~Pb}_{x} \mathrm{Se}_{1-y} \mathrm{~S}_{y}$ system.

while the number in the other concentrations $y$ is not specified even though the temperature is kept constant because of the component of 3 in the quaternary system.

Table 1 shows the compositional dependence of the lattice constant within the solubility range at the fixed composition $y$. There can be seen the positive inclination of the lattice constant with respect to $x$ in all cases, and also seen the tendency to decrease in the section of the lattice constant at $x=0$ with decreasing $y$. The facts that the addition of $\mathrm{PbSe}$ to CaSe causes the increase of the lattice constant, while the addition of $\mathrm{CaS}$ to $\mathrm{CaSe}$ causes its decrease are obviously due to the differences in the ionic radii between $\mathrm{Ca}$ and $\mathrm{Pb}$ and between $\mathrm{Se}$ and $\mathrm{S}$, respectively.

Figure 4 shows the solubility range and lattice constant of the $\mathrm{Ca}_{1-x} \mathrm{~Pb}_{x} \mathrm{Se}_{1-y} \mathrm{~S}_{y}$ system. The dashed line indicates the isolattice-matched line, and the solid line indicates the solubility limit at $1273 \mathrm{~K}$ obtained from the variation of the lattice constants (Fig. 3). The relations of lattice-matched to $\mathrm{PbS}$ and $\mathrm{InP}$ are also represented by the dotted line in the same figure. The solubility limit at $1273 \mathrm{~K}$ varies with respect to the Se concentration $y$, being resulted in minimum limit of 0.04 at $y=0.8$ in contrast to a maximum limit of 0.24 at $y=0$. From the results shown in Figs. 3 and 4, it is seen that the lattice constant of the $\mathrm{Ca}_{1-x} \mathrm{~Pb}_{x} \mathrm{Se}_{1-y} \mathrm{~S}_{y}$ system varies continuously from $0.5696(\mathrm{CaS}) \mathrm{nm}$ to $0.5993 \mathrm{~nm}$ $\left(\mathrm{Ca}_{0.76} \mathrm{~Pb}_{0.24} \mathrm{Se}\right)$, and it is clear that the $\mathrm{Ca}_{1-x} \mathrm{~Pb}_{x} \mathrm{Se}_{1-y} \mathrm{~S}_{y}$ system can be lattice-matched to $\mathrm{PbS}$ and $\mathrm{InP}$, which will be employed as substrate in order to fabricate the devices operated through intersubband transition.

Figure 5 shows the relationship of the solubility limits in the $\mathrm{Ca}_{1-x} \mathrm{~Pb}_{x} \mathrm{Se}_{1-y} \mathrm{~S}_{y}$ system with respect to the constituent

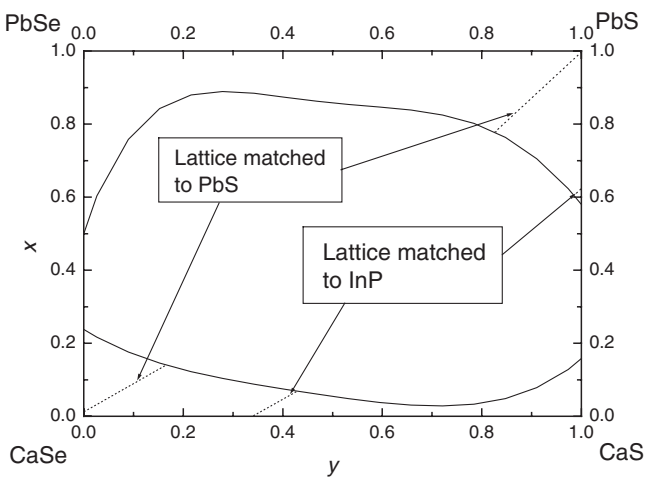

Fig. 5 Relationships of solubility limits in $\mathrm{Ca}_{1-x} \mathrm{~Pb}_{x} \mathrm{Se}_{1-y} \mathrm{~S}_{y}$ system with respect to the constituent elements of $\mathrm{PbSe}, \mathrm{PbS}, \mathrm{CaSe}$ and $\mathrm{CaS}$. The data in $\mathrm{Pb}$ rich region has been referred from our previous result. ${ }^{7}$ Relationships of lattice-matched to $\mathrm{PbS}$ and $\mathrm{InP}$ are also shown.

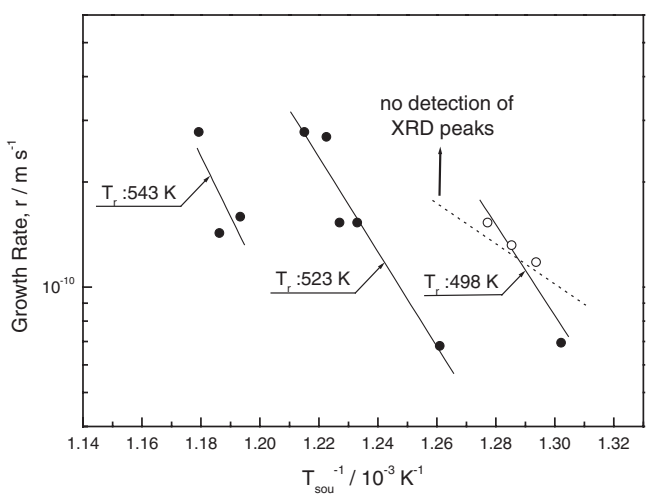

Fig. 6 Growth rate of the CaSe thin films a function of inverse of the source temperature.

elements of $\mathrm{PbSe}, \mathrm{PbS}, \mathrm{CaSe}$ and $\mathrm{CaS}$, by combining the present result for $\mathrm{Ca}$ rich region and the previous result for $\mathrm{Pb}$ rich region. ${ }^{7)}$ The relation of lattice-matched to $\mathrm{PbS}$ and $\mathrm{InP}$ is also shown with the dotted lines. It is seen from the figure that the isolattice-matched lines to $\mathrm{PbS}$ and $\mathrm{InP}$ exist in both $\mathrm{Pb}$ rich region (upper part in the figure) and $\mathrm{Ca}$ rich region (lower part), respectively. This implies that a lattice-matched heterostructure constructed by layering alternately $\mathrm{Ca}$ rich wide-gap compound and $\mathrm{Pb}$ rich narrow-gap compound is expected to give rise to a large difference in energy band gap. A numerical estimation of the difference will be performed in the following section, since the energy band gap at RT of CaSe has not only been reported yet among these constituent elements.

Next, we shall investigate CaSe thin films as a first step for preparing the quaternary thin films. Figure 6 shows the growth rate of the CaSe thin films as a function of inverse of the source temperature $\left(T_{\text {sou }}\right)$ under the reservoir temperature $\left(T_{\mathrm{r}}\right)$ of 498,523 and $543 \mathrm{~K}$. The substrate temperature $\left(T_{\text {sub }}\right)$ was kept constant at $873 \mathrm{~K}$. The plots of $\bigcirc$ and $\bigcirc$ mean detection and no detection of XRD peaks respectively within the precision of our experimental technique. The films with no detection of XRD peaks has brown-colored appearance in contrast to the colorless appearance in other films, indicating no sign of surface degradation for storage in ambient air. As can be seen in the figure, CaSe films with no XRD peaks 


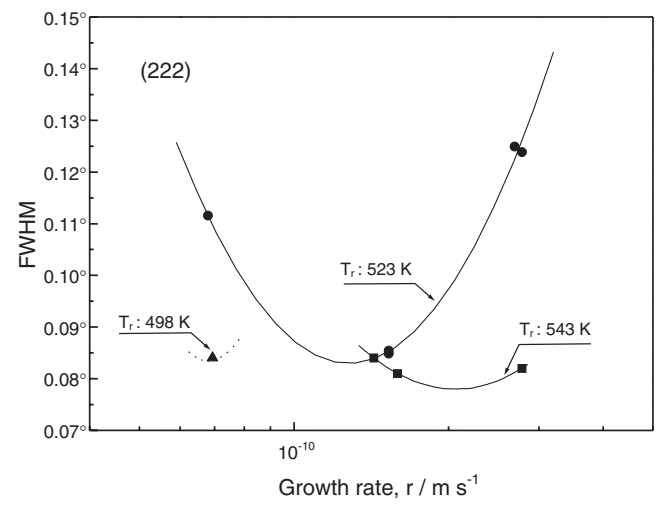

Fig. 7 The full-width at half maximum (FWHM) of X-ray rocking curve by $\omega-2 \theta$ scan as a function of growth rate.

seems to be appeared at the relatively low $T_{\mathrm{r}}$ of $498 \mathrm{~K}$. It is responsible for the fact that the Se vapor flux from the reservoir might be insufficient so as to construct well diffracting CaSe compound, suggesting considerable deviation from the appropriate ratio of $\mathrm{Se} / \mathrm{Ca}$ vapor flux. The growth rate clearly depends on the $T_{\text {sou }}$, so that growth process of the CaSe thin films is limited by the sublimation of Ca resource, being estimated activation energy to be $113 \mathrm{~kJ} /$ mol at $T_{\mathrm{r}}$ of $523 \mathrm{~K}$ assuming linear variation. It is also clear from the figure that the amount of $\mathrm{Ca}$ deposited on the substrate would be decreased with increasing the Se vapor flux when the $T_{\text {sou }}$ will be kept constant, since the linear variation of the growth rate shifts toward higher $T_{\text {sou }}$ as increasing $T_{\mathrm{r}}$. Accordingly, it is found that the vaporization from $\mathrm{Ca}$ resource is strongly affected by that from $\mathrm{Se}$ resource in the hot-wall epitaxy method.

Figure 7 shows the full-width at half-maximum (FWHM) of X-ray rocking curve by $\omega-2 \theta$ scan at (222) as a function of the growth rate. As seen in the figure, the FWHM seems to be varied parabolically with respect to the growth rate, being caused by change in vapor flux ratio of Se/Ca. Namely, at $T_{\mathrm{r}}$ of $523 \mathrm{~K}$, for example, FWHM comes to a minimum in the vicinity of $1.27 \times 10^{-10} \mathrm{~m} \mathrm{~s}^{-1}$, meaning that an appropriate vapor flux ratio of $\mathrm{Se} / \mathrm{Ca}$ is realized at the growth rate, while a growth rate higher than $1.27 \times 10^{-10} \mathrm{~m} \mathrm{~s}^{-1}$ brings $\mathrm{Ca}$ excess deviation from the appropriate ratio, and a lower growth rate leads to Se excess deviation. The appropriate ratio, however, cannot be evaluated numerically in the present study because of no monitoring system of these vapor fluxes in the apparatus used. The parabolic variation shift toward higher growth rate as increasing the $T_{\mathrm{r}}$, and, at $T_{\mathrm{r}}$ of $543 \mathrm{~K}$, the CaSe thin film has the FWHM of $0.08^{\circ}$ by $\omega-2 \theta$ scan and $0.29^{\circ}$ by $\omega$ scan, which is the best crystallinity obtained in the present study. A FWHM of the diffractogram obtained by $\omega$ scan reflects a degree of mosaicity of a crystal in contrast to unevenness of the lattice constant by $\omega-2 \theta$ scan. Therefore, the quality of the CaSe thin films is limited by mosaicity rather than unevenness of lattice constant. The considerable large FWHM by $\omega$ scan compared to that by $\omega-2 \theta$ scan would be attributed to lattice misfit of $4.45 \%$ between $\mathrm{CaSe}$ and $\mathrm{BaF}_{2}$ substrate.

Figure 8 shows typical XRD pattern of CaSe thin films at $T_{\text {sub }}$ of $873 \mathrm{~K}$ by $\omega-2 \theta$ scan (a) and $\phi$-scan (b). As can be seen
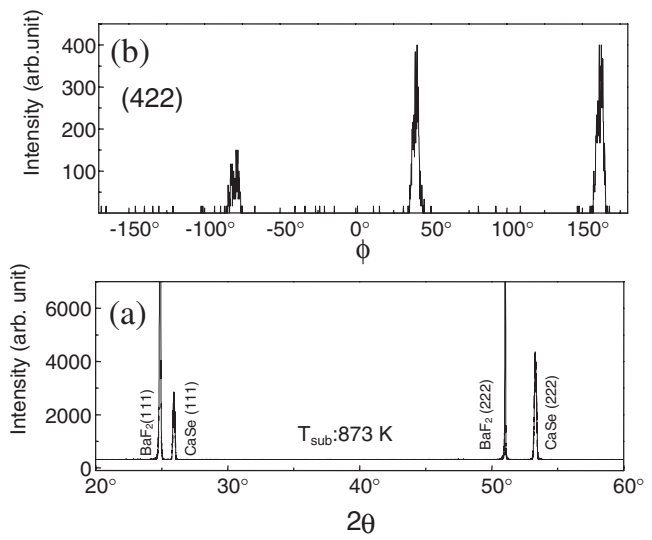

Fig. 8 Typical XRD pattern of CaSe thin films at $T_{\text {sub }}$ of $873 \mathrm{~K}$ by $\omega-2 \theta$ scan (a) and $\phi$-scan (b).

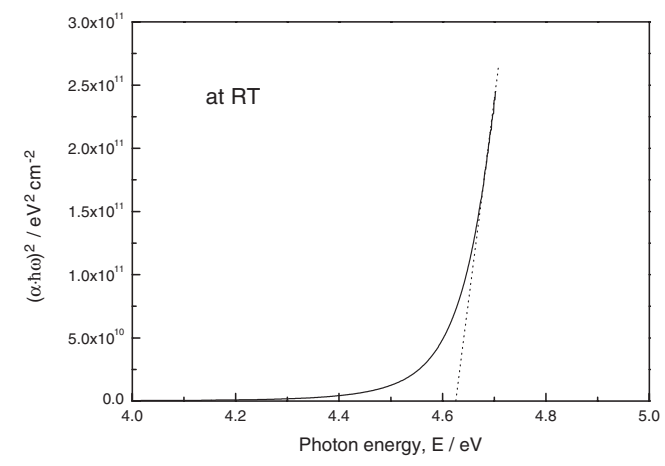

Fig. 9 Typical absorption edge at RT of the CaSe thin film.

in the patterns (a) that (111) and (222) peaks of CaSe thin film with $\mathrm{NaCl}$ structure are observed together with the peaks of $\mathrm{BaF}_{2}$ substrate, taking the growth orientation at [111] direction on cleaved $\mathrm{BaF}_{2}(111)$ substrate. Similar patterns have been observed on the thin films grown at the $T_{\text {sub }}$ range from 673 to $873 \mathrm{~K}$. We also investigated the in-plane texture of the film by azimuthal $\phi$-scan (b). Every $120^{\circ}$ of these reflections confirm that the film contains a single cubic texture in the film plane. Therefore, epitaxial growth of the CaSe thin film can be successfully performed employing the hot-wall epitaxy. Moreover, FWHM of the X-ray rocking curve at (222) by $\omega$ scan decreased monotonically with increasing the $T_{\text {sub }}$. Thus, crystallinity of the CaSe thin films can be improved with respect to the $T_{\text {sub }}$.

So far, optical absorption at $77 \mathrm{~K}$ of CaSe thin films deposited on quartz plate ${ }^{11)}$ and optical reflectivity at $2 \mathrm{~K}$ of CaSe single crystal $^{12)}$ have been reported, observing several energy peaks of 4.87 and $5.11 \mathrm{eV}$ in the former, and of 4.828, 5.056 and $5.60 \mathrm{eV}$ in the latter. Optical energy band gap, however, have not been estimated experimentally through optical absorption coefficient. Then, we have tried to estimate the energy band gap at RT of the CaSe thin film grown epitaxially. Figure 9 shows the typical absorption edge at RT of the CaSe thin film grown at the $T_{\text {sub }}$ of $873 \mathrm{~K}$. The absorption coefficient $\alpha$ of the CaSe thin film was obtained through the measurement of transmittance and reflectance of the film, employing the following expression: $\alpha \mathrm{d}=$ $\ln \left(T_{\mathrm{obs}}{ }^{-1}\right)+\ln \left(1-R_{\mathrm{obs}}\right)+\ln \left(1-R_{\mathrm{obs}}^{\prime}\right)+\ln \left(1-R_{\mathrm{obs}}^{\prime}+R_{\mathrm{sobs}} /\right.$ 


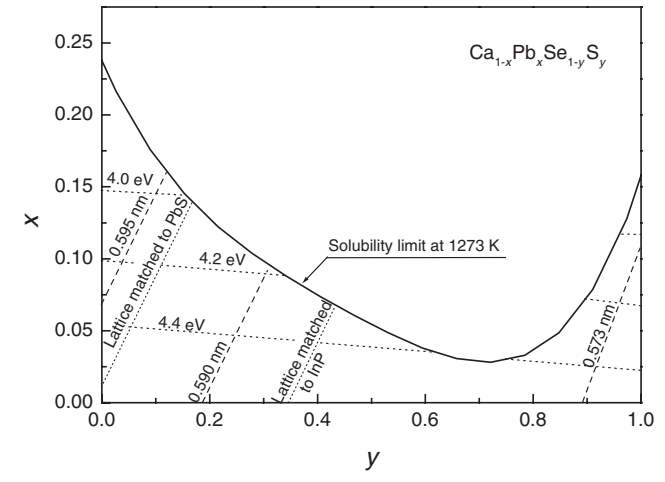

Fig. $10 x-y$ compositional plane of the $\mathrm{Ca}_{1-x} \mathrm{~Pb}_{x} \mathrm{Se}_{1-y} \mathrm{~S}_{y}$ system.

$2),{ }^{13)}$ where $\mathrm{d}$ is the thickness of the film, $T_{\mathrm{obs}}$ is transmittance, $R_{\mathrm{obs}}$ is reflectance observed at film-side and $R_{\mathrm{obs}}^{\prime}$ is reflectance observed at substrate-side. So that, the energy band gap is estimated to be $4.62 \mathrm{eV}$ by linear extrapolation to zero of the $\alpha \cdot \hbar \omega$ squared. In addition, no energy peaks are observed at the photon energy range above the optical absorption edge.

Figure 10 shows $x-y$ compositional plane of the $\mathrm{Ca}_{1-x} \mathrm{~Pb}_{x} \mathrm{Se}_{1-y} \mathrm{~S}_{y}$ system. The isoenergy band gap lines with respect to the compositions in $\mathrm{Ca}_{1-x} \mathrm{~Pb}_{x} \mathrm{Se}_{1-y} \mathrm{~S}_{y}$ system are estimated using the following expressions: $E g=4.62-$ $4.21 x-0.12 y-0.02 x y$, where linear variation of the energy band gap between the constituent elements is assumed as first-approximation, employing $4.62 \mathrm{eV}$ for $\mathrm{CaSe}, 4.5 \mathrm{eV}$ for $\mathrm{CaS},{ }^{14)} 0.27 \mathrm{eV}$ for $\mathrm{PbSe}^{15)}$ and $0.41 \mathrm{eV}$ for $\mathrm{PbS}{ }^{15)}$ As a result of obtaining the energy band gap of CaSe, relation between lattice constant and energy band gap of $\mathrm{Ca}_{1-x} \mathrm{~Pb}_{x} \mathrm{Se}_{1-y} \mathrm{~S}_{y}$ system can be discussed. In general, energy band gap of ternary- or quaternary-system with miscibility gap varies nonlinearly with respect to composition. In the present study, it is anticipated that $\mathrm{Ca}_{1-x} \mathrm{~Pb}_{x} \mathrm{Se}_{1-y} \mathrm{~S}_{y}$ system has also nonlinear variation of energy band gap with respect to $\mathrm{Pb}$ content. It is found from the figure that there can be considered $\mathrm{Ca}_{0.99} \mathrm{~Pb}_{0.01} \mathrm{Se} / \mathrm{PbS}$ - or $\mathrm{CaSe}_{0.66} \mathrm{~S}_{0.34} / \mathrm{InP}$-heterostructure, for example, in case of employing ternary compound. We can see that large band gap difference of about 4 or $3 \mathrm{eV}$ is possibly obtained in $\mathrm{Ca}_{0.99} \mathrm{~Pb}_{0.01} \mathrm{Se} / \mathrm{PbS}$ or $\mathrm{CaSe}_{0.66} \mathrm{~S}_{0.34} / \mathrm{InP}$. We should pay attention to the substantially large difference in energy band gap under keeping lattice-match condition with the substrate, suggesting the $\mathrm{Ca}_{1-x} \mathrm{~Pb}_{x} \mathrm{Se}_{1-y} \mathrm{~S}_{y}$ system to have considerable promise as a material for devices through intersubband transition. We need further investigations concerning the bowing parameter in the variation of energy band gap and the type of band alignment in these heterostructure through preparation of ternary or quaternary thin film growth.

\section{Conclusion}

It may be remarked that the $\mathrm{Ca}_{1-x} \mathrm{~Pb}_{x} \mathrm{Se}_{1-y} \mathrm{~S}_{y}$ system is regarded as good candidate materials for producing compounds to construct the devices to be operated through intersubband transition. $\mathrm{Ca}_{1-x} \mathrm{~Pb}_{x} \mathrm{Se}_{1-y} \mathrm{~S}_{y}$ system has adequate solubility limit at $1273 \mathrm{~K}$ to form such solid solution semiconductor, being lattice-matched to $\mathrm{PbS}$ and $\mathrm{InP}$ used as substrate. It would be also pointed out that epitaxial growth of CaSe thin films are successfully performed through the $T_{\text {sub }}$ range between 673 and $873 \mathrm{~K}$ by means of hot-wall epitaxy. The energy band gap of the CaSe is found to be $4.62 \mathrm{eV}$ at RT optical absorption spectrum. Based on the results obtained in the present study, ternary and quaternary CaSe-based solid solution thin films will be fabricated in our subsequent investigation, expecting to bring advanced optical devices.

\section{REFERENCES}

1) C. Gmachi, H. M. Ng, S.-N. G. Chu and A. y. Cho: Appl. Phys. Lett. 77 (2000) 3722-3724.

2) B. Sung, H. C. Chui, M. N. Fejer and J. S. Harris, Jr.: Electron. Lett. 33 (1997) 818-820.

3) T. Mozume, H. Yoshida, A. Neogi and M. Kudo: Jpn. J. Appl. Phys. 38 (1999) 1286-1289.

4) R. Akimoto, Y. Kinpara, K. Akita, F. Sasaki and S. Kobayashi: Appl. Phys. Lett. 78 (2001) 580-582.

5) Y. Nakata, Y. Sugiyama, T. Inata, O. Ueda, S. Sasa, S. Muto and T. Fujii: MRS Symp. Proc. 198 (1990) 289-294.

6) $1 \mathrm{eV}=1.60218 \times 10^{-19} \mathrm{~J}$

7) S. Abe and K. Masumoto: J. Crystal Growth 204 (1999) 115-121.

8) R. Pandey, J. E. Jaffe and B. Kunz: Phys. Rev. B 43 (1991) 9228-9237.

9) J. B. Nelson and D. P. Riley: Proc. Phys. Soc. 57 (1945) 160-177.

10) L. Vegard: Z. Phys. 5 (1921) 17-26.

11) G. A. Saum and E. B. Hensley: Phys. Rev. 115 (1959) 1019-1022.

12) Y. Kaneko and T. Koda: J. Crystal Growth 86 (1988) 72-78.

13) S. Yoshida and H. Yajima: Optical thin films and devices, (Univ. of Tokyo Press, Tokyo, 1994) pp. 73.

14) N. Yamashita: Jpn. J. Appl. Phys. 30 (1991) 1384-1485.

15) J. N. Zemel, J. D. Jensen and R. B. Schoolar: Phys. Rev. 140 (1965) A330-A342. 